\title{
Coordinate-space calculation of radiative corrections to the B-meson distribution amplitude: light-cone vs. static distributions
}

\author{
Hiroyuki Kawamura*† \\ Department of Mathematics, Juntendo University, Inzai, Chiba 270-1695, Japan \\ E-mail: h-kawamura@juntendo.ac.jp

\section{Kazuhiro Tanaka} \\ Department of Physics, Juntendo University, Inzai, Chiba 270-1695, Japan and \\ J-PARC Branch, KEK Theory Center, Institute of Particle and Nuclear Studies, KEK, 203-1, \\ Shirakata, Tokai, Ibaraki, 319-1106, Japan \\ E-mail: kztanaka@juntendo.ac.jp
}

\begin{abstract}
We discuss the relation between the light-cone and static distributions for the B meson in the framework of the heavy quark effective theory. We investigate the possibility of calculating the B-meson light-cone distribution by lattice QCD applying the recently proposed Ji's approach. We discuss the tree-level relations between the light-cone and static distributions and calculate the 1-loop corrections to them in the $\overline{\mathrm{MS}}$ scheme using the background field method in the coordinate space.
\end{abstract}

13th International Symposium on Radiative Corrections 24-29 September, 2017

St. Gilgen, Austria

\footnotetext{
* Speaker.

† Supported by JSPS KAKENHI Grant Number JP15K0506.

${ }^{\ddagger}$ Supported by JSPS KAKENHI Grant Numbers JP25610058 and JP26287040.
} 


\section{Introduction}

Heavy flavor physics provides one of the promising way to explore physics beyond the Standard Model. The indirect searches for New Physics via precision measurements are complementary to the direct searches at collider experiments such as LHC. The BELLE II experiment at the SuperKEKB, whose expected integrated luminocity is as much as $50 \mathrm{ab}^{-1}$, will start its data taking in late 2018 [1], where the various decay modes of heavy hadrons are measured with the unprecedented accuracy. In order to make full use of the BELLE II data, it is important to make the theoretical predictions as reliable as possible for the decay channels to be measured.

One of the rare decays of the B meson which is expected to be measured at BELLE II is the leptonic radiative decays: $B \rightarrow \gamma l v$. The amplitude of this process can be expressed by the factorization formula as

$$
\mathscr{A}(B \rightarrow \gamma l v) \propto \int_{0}^{\infty} \frac{d \omega}{\omega} T\left(\omega, m_{b}, \mu ; \alpha_{s}\right) \phi_{+}^{B}(\omega, \mu)+\mathscr{O}\left(1 / m_{b}\right),
$$

when the emitted photon is energetic [2]. The hard coefficient $T\left(\omega, m_{b}, \mu ; \alpha_{s}\right)$ is perturbatively calculable and the light-cone distribution amplitude (LCDA) of B-mesons is defined as [3, 4]

$$
\phi_{+}^{B}(\omega, \mu)=\frac{1}{i F(\mu)} \int_{-\infty}^{\infty} \frac{d t}{2 \pi} e^{i \omega t}\left\langle 0\left|\bar{q}(t n) h \gamma_{5}[t n, 0] h_{v}(0)\right| \bar{B}(v)\right\rangle,
$$

for $\omega>0$, where the bi-local operator is defined by the light-antiquark and b-quark fields, $\bar{q}(t n)$ and $h_{v}(0)$, linked by the Wilson line $[t n, 0]$ with a light-like separation $t n^{\mu}$, using a light-like vector $n^{\mu}$, and $v^{\mu}(n \cdot v=1)$ is the 4-velocity of the B-meson. The B-meson LCDA of (1.2) is defined in the heavy quark effective theory (HQET), where $h_{v}(x)$ and $F(\mu)$ are the HQET field and decay constant, respectively. The variable $\omega$ parametrizes the LC component of the light-antiquark's momentum inside the B meson as $k^{+}=\omega v^{+}$. The factorization formula (1.1) is proportional to the inverse moment of the LCDA, $\frac{1}{\lambda_{B}(\mu)} \equiv \int_{0}^{\infty} \frac{d \omega}{\omega} \phi_{+}(\omega, \mu)$ at the leading order, and is expressed by $\frac{1}{\lambda_{B}(\mu)}$ and $\frac{\sigma_{n}(\mu)}{\lambda_{B}(\mu)} \equiv \int_{0}^{\infty} \frac{d \omega}{\omega}\left(\ln \frac{\mu}{\omega}\right)^{n} \phi_{+}(\omega, \mu)(n=1,2)$ at the next-to leading order [3]. These quantities are among the important nonperturbative inputs for the phenomenology not only of the leptonic radiative decays but also of the non-leptonic exclusive decays of B-mesons [5]. Therefore, precise knowledge of these quantities is crucial for extracting the CP phases from the measurements of CP asymmetries in those processes.

The B-meson LCDA has been studied by various authors. Some model DAs are proposed based on the QCD sum rules [3], and relations among two- and three-body LCDAs of B-mesons are discussed in [4]. The scale evolution of the B-meson LCDA has been studied by various authors [6]. What is characteristic of the B-meson LCDA is that its positive moments are not well-defined due to the hard radiative tail [6]. Namely, the logarithmic terms $\propto \ln ^{n} \frac{\omega}{\mu}$ appear in the radiative corrections, so that the positive moments are divergent in contrast to the light-meson cases. Therefore, the relation between the bi-local operator in (1.2) and the corresponding local operators is given via the operator product expansion with the Wilson coefficients which contain the logarithmic terms. The 1-loop matching has been calculated up to the dimension-4 [7] and dimension-5 [8] local operators to construct a model of the B-meson LCDA. 
Recently, Ji proposed a novel approach to calculate hadron matrix elements of bi-local lightcone operators directly by lattice QCD [9]. The idea is that the light-cone distributions are reproduced by the static distributions in large $P^{z}$ limit of hadron momentum. Since the original Ji's paper, there have been many studies, which are mainly for the parton distribution functions (PDFs). Some preliminary results of lattice simulation have already been presented for the non-singlet quark PDFs by some groups [10]. In this work, we consider the possibility of evaluating the B-meson LCDA by lattice QCD. Firstly, we discuss the relation between the light-cone and static distributions for the B-meson, and then calculate the 1-loop corrections to them.

\section{Light-cone vs. static correlations}

In this section, we review the Ji's argument to calculate the quark PDFs by lattice QCD and discuss how the argument is modified in the case of the B-meosn LCDA. The (unpolarized) quark PDF is defined as a light-like correlation as

$$
q\left(x, \mu^{2}\right)=\int \frac{d \xi^{-}}{4 \pi} e^{-i x P^{+} \xi^{-}}\left\langle P\left|\bar{q}\left(\xi^{-}\right) \gamma^{+}\left[\xi^{-}, 0\right] q(0)\right| P\right\rangle .
$$

Since the light-cone correlation depends on the real time, it cannot be calculated directly by lattice QCD. On the other hand, the quark "quasi-PDF" defined by the static correlation,

$$
\tilde{q}\left(x, \mu^{2}, P^{z}\right)=\int \frac{d z}{4 \pi} e^{i x P^{z} z}\left\langle P\left|\bar{q}(z) \gamma^{z}[z, 0] q(0)\right| P\right\rangle,
$$

is calculable on the lattice. The relation between these distributions can be seen by comparing the matrix elements of the corresponding local operators. The matrix elements of the twist- 2 operators for the PDF are given by [11]

$$
\left\langle P\left|\bar{q} \gamma^{+}\left(i D^{+}\right)^{n-1} q\right| P\right\rangle=2 a_{n}\left(\mu^{2}\right) \times\left(P^{+}\right)^{n},
$$

while those for the quasi-PDF are

$$
\left\langle P\left|\bar{q} \gamma^{z}\left(i D^{z}\right)^{n-1} q\right| P\right\rangle=2 a_{n}\left(\mu^{2}\right) \times\left(P^{z}\right)^{n} \times\left[1+\mathscr{O}\left(\Lambda_{\mathrm{QCD}}^{2} /\left(P^{z}\right)^{2}, M^{2} /\left(P^{z}\right)^{2}\right)\right],
$$

where $a_{n}\left(\mu^{2}\right)$ is the reduced matrix elements of the twist-2 operators. In (2.4), the power corrections in the parentheses correspond to the higher-twist and target-mass effects. These expressions suggest that the quasi-PDF is related to the PDF as

$$
\tilde{q}\left(x, \mu^{2}, P^{z}\right)=q\left(x, \mu^{2}\right)\left[1+\mathscr{O}\left(\Lambda_{\mathrm{QCD}}^{2} /\left(P^{z}\right)^{2}, M^{2} /\left(P^{z}\right)^{2}\right)\right],
$$

so that they coincide in the large $P^{z}$ limit. Actually, we should consider this relation to hold only at the tree level and, when the radiative corrections are taken into account, this relation would be modified and expressed in a factorization formula [9]:

$$
\tilde{q}\left(x, \mu^{2}, P^{z}\right)=\int \frac{d y}{y} Z\left(\frac{x}{y}, \frac{\mu}{P^{z}} ; \alpha_{s}\right) q\left(y, \mu^{2}\right)+\mathscr{O}\left(\Lambda_{\mathrm{QCD}}^{2} /\left(P^{z}\right)^{2}, M^{2} /\left(P^{z}\right)^{2}\right) .
$$

The matching coefficient $Z$ has been calculated by many authors, and the results are given in various regularization and renormalization schemes [9,12]. 
In the present case of the B-meson LCDA, we consider the following quantity as the "quasiDA" of the B-meson:

$$
\Psi_{+}^{B}\left(\omega, \mu, v^{z}\right)=\frac{1}{i F(\mu)} \int_{-\infty}^{\infty} \frac{d z}{2 \pi} e^{-i \omega v^{z} z}\left\langle 0\left|\bar{q}(z) \notin \gamma_{5}[z, 0] h_{v}(0)\right| \bar{B}(v)\right\rangle .
$$

Here the 4-velocity of the B meson is taken to be $v^{\mu}=\left(\frac{1}{\sqrt{1-\beta^{2}}}, 0,0, \frac{\beta}{\sqrt{1-\beta^{2}}}\right)$. The tree level relation between the light-cone distribution of (1.2) and the static distribution of (2.7) can be read from the matrix elements of the corresponding local operators:

$$
\left\langle 0\left|\bar{q} \gamma^{+} \gamma^{5}\left(i \overleftrightarrow{D}^{+}\right)^{n-1} h_{v}\right| B(v)\right\rangle=i F(\mu) \Theta_{n}\left(\mu^{2}\right) \times\left(v^{+}\right)^{n}
$$

and

$$
\left\langle 0\left|\bar{q} \gamma^{z} \gamma_{5}\left(i \overleftrightarrow{D}^{z}\right)^{n-1} h_{v}\right| B(v)\right\rangle=i F(\mu) \Theta_{n}\left(\mu^{2}\right) \times\left(v^{z}\right)^{n} \times\left[1+\mathscr{O}\left(\frac{1}{\left(v^{z}\right)^{2}}\right)\right]
$$

These matrix elements suggest the tree level relation, $\Psi_{+}^{B}\left(\omega, \mu, v^{z}\right)=\phi_{+}^{B}(\omega, \mu)\left[1+\mathscr{O}\left(1 /\left(v^{z}\right)^{2}\right)\right]$, which is similar to the tree-level relation (2.5) for the quark PDF and quasi-PDF, with $P^{z}$ replaced by $v^{z}$. In the present case, both the higher-twist and target mass effects are $\mathscr{O}\left(1 /\left(v^{z}\right)^{2}\right)$ corrections due to the lack of large mass scales in the HQET. As in the case of the quark PDF, the UV structures of the quasi-DA and LCDA of B-meson would be different; furthermore, as mentioned below (1.2) in Sec. 1, the B-meson LCDA is expressed as the operator product expansion in terms of the local operators (2.8) combined with the Wilson coefficients that exhibit the $\ln ^{n} \omega$ behavior corresponding to the hard radiative tail, and similarly for the quasi-DA in terms of the local operators 2.96) combined with the corresponding Wilson coefficients. Therefore, the relation between them should be given as the factorization formula,

$$
\Psi_{+}^{B}\left(\omega, \mu, v^{z}\right)=\int d \omega^{\prime} C\left(\omega, \omega^{\prime}, \ln \left(\mu / \omega v^{z}\right) ; \alpha_{s}\right) \phi_{+}^{B}\left(\omega^{\prime}, \mu\right)+\mathscr{O}\left(\frac{1}{\left(v^{z}\right)^{2}}\right) .
$$

This means that the quasi-DA of (2.7) can be related to the LCDA of (1.2) in the large $v^{z}$ limit.

\section{1-loop corrections}

In this section, we calculate the 1-loop corrections to the bi-local operator with a space-like separation, $\bar{q}(\xi) \xi \gamma_{5}[\xi, 0] h_{v}(0)\left(\xi^{2}<0\right)$, and compare them with the corrections to a light-like operator, $\bar{q}(t n) h \gamma_{5}[t n, 0] h_{v}(0)$. The diagrams (a)-(d) in Fig.1 contribute to the space-like operator, while the diagram $(\mathrm{d})$ vanishes for the light-like operator. The calculation is done in the coordinate space using the background field method. The Fock-Schwinger gauge, $\xi \cdot A^{c l}(\xi)=0$, is taken for the classical gluon field, and the heat kernel expansion was performed to extract the leading power contributions [13].

The corrections to the space-like and light-like operators coming from the diagram (a) are

$$
\begin{aligned}
{\left[\bar{q}(\xi) \xi \gamma_{5}[\xi, 0] h_{v}(0)\right]^{(a)}=} & -\frac{\alpha_{s}}{4 \pi} C_{F}\left[\frac{1}{\varepsilon_{\mathrm{UV}}}\left\{2 \ln [2 i \tilde{\mu}(\xi \cdot v)]-\ln \left(-\tilde{\mu}^{2} \xi^{2}\right)\right\}\right. \\
& \left.+2 \ln ^{2}[2 i \tilde{\mu}(\xi \cdot v)]-\frac{1}{2} \ln ^{2}\left(-\tilde{\mu}^{2} \xi^{2}\right)+\frac{\pi^{2}}{3}\right] \bar{q}(\xi) \xi \gamma_{5}[\xi, 0] h_{v}(0),
\end{aligned}
$$




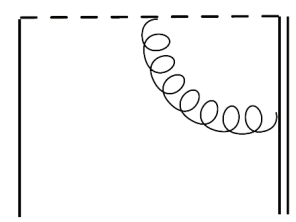

(a)

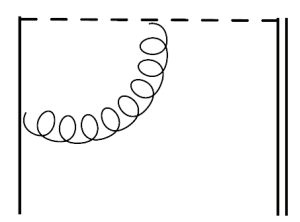

(b)

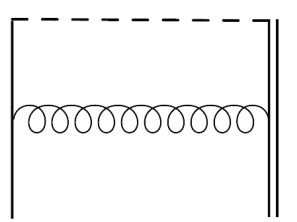

(c)

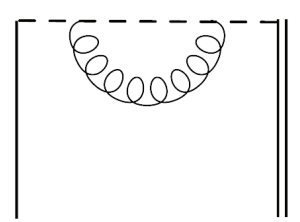

(d)

Figure 1: 1-loop corrections to the quasi-distribution of B meson. The double and dashed lines are the HQET field and Wilson line, respectively.

and

$$
\left[\bar{q}(t n) h \gamma_{5}[t n, 0] h_{v}(0)\right]^{(a)}=-\frac{\alpha_{s}}{4 \pi} C_{F}\left[\frac{1}{\varepsilon_{\mathrm{UV}}^{2}}+\frac{2 \ln (2 i \tilde{\mu} t)}{\varepsilon_{\mathrm{UV}}}+2 \ln ^{2}(2 i \tilde{\mu} t)+\frac{5}{12} \pi^{2}\right] \bar{q}(t n) \hbar \gamma_{5}[t n, 0] h_{v}(0),
$$

respectively, where $D=4-2 \varepsilon_{\mathrm{UV}}$ is the space-time dimension, $C_{F}=\left(N_{C}^{2}-1\right) /\left(2 N_{C}\right)$ and $\tilde{\mu}=$ $\sqrt{\pi} \mu e^{\gamma_{E} / 2}$. The higher twist operators are dropped in the right-hand sides because those contributions are suppressed by $1 /\left(v^{z}\right)^{2}$. The UV poles are related to the cusp singularities which generally appear in the vertex-type corrections to a product of two Wilson lines, and the logarithmic terms $\ln \left(-\tilde{\mu}^{2} \xi^{2}\right)$ and $\ln ^{2}\left(-\tilde{\mu}^{2} \xi^{2}\right)$ in 3.10 correspond to the light-cone singularities. From the diagram (b), we obtain

$$
\begin{aligned}
{\left[\bar{q}(\xi) \xi \gamma_{5}[\xi, 0] h_{v}(0)\right]^{(b)}=\frac{\alpha_{s}}{2 \pi} C_{F} } & {\left[\frac{1}{2}\left\{\frac{1}{\varepsilon_{\mathrm{UV}}}+\ln \left(-\tilde{\mu}^{2} \xi^{2}\right)\right\} \bar{q}(\xi) \xi \gamma_{5}[\xi, 0] h_{v}(0)\right.} \\
& -\left\{\frac{1}{\varepsilon_{\mathrm{IR}}}+\ln \left(-\tilde{\mu}^{2} \xi^{2}\right)\right\} \int_{0}^{1} d u \frac{u}{1-u}[\bar{q}(u \xi)-\bar{q}(\xi)] \xi \gamma_{5}[\xi, 0] h_{v}(0) \\
& \left.-\int_{0}^{1} d u \frac{2 \ln (1-u)+u}{1-u}[\bar{q}(u \xi)-\bar{q}(\xi)] \xi \gamma_{5}[\xi, 0] h_{v}(0)\right]
\end{aligned}
$$

and

$$
\left[\bar{q}(t n) h \gamma_{5}[t n, 0] h_{v}(0)\right]^{(b)}=\frac{\alpha_{s}}{2 \pi} C_{F}\left(\frac{1}{\varepsilon_{\mathrm{UV}}}-\frac{1}{\varepsilon_{\mathrm{IR}}}\right) \int_{0}^{1} d u \frac{u}{1-u}[\bar{q}(v t n)-\bar{q}(t n)] h \gamma_{5}[t n, 0] h_{v}(0) .
$$

The coefficient of the IR poles in (3.3) and (3.4) is what appears in the calculation of the BrodskyLepage kernel for the light-meson LCDAs [14]. Then the diagram (c) gives

$$
\left[\bar{q}(\xi) \xi \gamma_{5}[\xi, 0] h_{v}(0)\right]^{(c)}=\frac{\alpha_{s}}{4 \pi} C_{F}\left\{-\frac{1}{\varepsilon_{\mathrm{IR}}}-2 \ln [2 i \tilde{\mu}(\xi \cdot v)]+2 \ln 2\right\} \int_{0}^{1} d u \bar{q}(u \xi) \xi \gamma_{5}[\xi, 0] h_{v}(0),
$$

up to the possible higher dimensional contributions which will be discussed elsewhere, and

$$
\left[\bar{q}(t n) h \gamma_{5}[t n, 0] h_{v}(0)\right]^{(c)}=\frac{\alpha_{s}}{4 \pi} C_{F}\left\{-\frac{1}{\varepsilon_{\mathrm{IR}}}-2 \ln (2 i \tilde{\mu} t)\right\} \int_{0}^{1} d u \bar{q}(u t n) h \gamma_{5}[t n, 0] h_{v}(0) .
$$


Finally, the diagram (d) gives

$$
\left[\bar{q}(\xi) \xi \gamma_{5}[\xi, 0] h_{v}(0)\right]^{(d)}=\frac{\alpha_{s}}{2 \pi} C_{F}\left\{\frac{1}{\varepsilon_{\mathrm{UV}}}+\ln \left(-\tilde{\mu}^{2} \xi^{2}\right)+2\right\} \bar{q}(\xi) \xi \gamma_{5}[\xi, 0] h_{v}(0) .
$$

From these results (3.1)-3.7), one can see that the IR structure is common between the spacelike and light-like operators, while the UV structure is different, which suggests that the matching of the form of (2.10) is adequate, and the 1-loop matching coefficient in the $\overline{\mathrm{MS}}$ scheme can be extracted. When the momentum cutoff is employed to regularize the UV divergence, the power divergences appear in the diagram (d). How to treat such power divergences in the quasi-PDF has been discussed in the context of the matching between the quasi-PDFs in the continuum and lattice QCDs [15]. We note that, in principle, the same discussion applies to the present case.

\section{Summary}

In this paper, the relation between the static and light-cone distributions for the heavy-light mesons was discussed in the heavy quark effective theory. We showed that the latter distribution is reproduced by the former in the large $v^{z}$ limit. Then we calculated the 1-loop correlations to the static and light-like operators in the $\overline{\mathrm{MS}}$ scheme. As for the feasibility of calculating the static distribution with the non-zero $v^{z}$ by lattice QCD, the moving NRQCD formalism may be useful [16]. Though a large numerical noise is anticipated, even an rough estimation of the B-meson LCDA would be helpful because we have almost no knowledge of the B-meson LCDA. For the practical usage of this approach, further studies are needed including the matching between the quasi-DA in the continuum and lattice QCD, which we leave for future work.

\section{References}

[1] R. de Sangro [BELLE-II Collaboration], PoS FPCP 2017 (2017) 037.

[2] G. P. Korchemsky, D. Pirjol and T. M. Yan, [hep-ph/9911427].

S. Descotes-Genon and C. T. Sachrajda, Nucl. Phys. B 650 (2003) 356 [hep-ph/0209216].

E. Lunghi, D. Pirjol and D. Wyler, Nucl. Phys. B 649 (2003) 349 [hep-ph/0210091].

S. W. Bosch, R. J. Hill, B. O. Lange and M. Neubert, Phys. Rev. D 67 (2003) 094014 [hep-ph/0301123].

M. Beneke and J. Rohrwild, Eur. Phys. J. C 71 (2011) 1818 [arXiv:1110.3228 [hep-ph]].

[3] A. G. Grozin and M. Neubert, Phys. Rev. D 55 (1997) 272 [hep-ph/9607366].

V. M. Braun, D. Y. Ivanov and G. P. Korchemsky, Phys. Rev. D 69 (2004) 034014 [hep-ph/0309330].

[4] M. Beneke and T. Feldmann, Nucl. Phys. B 592 (2001) 3 [hep-ph/0008255].

H. Kawamura, J. Kodaira, C. F. Qiao and K. Tanaka, Phys. Lett. B 523 (2001) 111 Erratum: [Phys. Lett. B 536 (2002) 344] [hep-ph/0109181].

[5] M. Beneke and M. Neubert, Nucl. Phys. B 675 (2003) 333 [hep-ph/0308039].

M. Beneke and S. Jager, Nucl. Phys. B 768 (2007) 51 [hep-ph/0610322].

[6] B. O. Lange and M. Neubert, Phys. Rev. Lett. 91 (2003) 102001 [hep-ph/0303082].

H. Kawamura and K. Tanaka, Phys. Rev. D 81 (2010) 114009 [arXiv:1002.1177 [hep-ph]].

G. Bell, T. Feldmann, Y. M. Wang and M. W. Y. Yip, JHEP 1311 (2013) 191 [arXiv:1308.6114 [hep-ph]].

B. O. Lange, Nucl. Part. Phys. Proc. 258-259 (2015) 171 [arXiv:1409.2438 [hep-ph]]. 
[7] S. J. Lee and M. Neubert, Phys. Rev. D 72 (2005) 094028 [hep-ph/0509350].

[8] H. Kawamura and K. Tanaka, Phys. Lett. B 673 (2009) 201 [arXiv:0810.5628 [hep-ph]].

[9] X. Ji, Phys. Rev. Lett. 110 (2013) 262002 [arXiv:1305.1539 [hep-ph]].

[10] H. W. Lin, J. W. Chen, S. D. Cohen and X. Ji, Phys. Rev. D 91 (2015) 054510 [arXiv:1402.1462 [hep-ph]].

C. Alexandrou, K. Cichy, V. Drach, E. Garcia-Ramos, K. Hadjiyiannakou, K. Jansen, F. Steffens and C. Wiese, Phys. Rev. D 92 (2015) 014502 [arXiv:1504.07455 [hep-lat]].

C. Alexandrou, K. Cichy, M. Constantinou, K. Hadjiyiannakou, K. Jansen, F. Steffens and C. Wiese, Phys. Rev. D 96 (2017) no.1, 014513 [arXiv:1610.03689 [hep-lat]]. H. W. Lin et al., arXiv:1711.07916 [hep-ph], and references therein.

[11] For example, J. Kodaira and K. Tanaka, Prog. Theor. Phys. 101 (1999) 191 [hep-ph/9812449].

[12] X. Xiong, X. Ji, J. H. Zhang and Y. Zhao, Phys. Rev. D 90 (2014) no.1, 014051 [arXiv:1310.7471 [hep-ph]].

Y. Q. Ma and J. W. Qiu, arXiv:1404.6860 [hep-ph].

I. W. Stewart and Y. Zhao, arXiv:1709.04933 [hep-ph].

[13] I. I. Balitsky and V. M. Braun, Nucl. Phys. B 311 (1989) 541.

[14] G. P. Lepage and S. J. Brodsky, Phys. Rev. D 22 (1980) 2157.

[15] T. Ishikawa, Y. Q. Ma, J. W. Qiu and S. Yoshida, arXiv:1609.02018 [hep-lat]. T. Ishikawa, Y. Q. Ma, J. W. Qiu and S. Yoshida, Phys. Rev. D 96 (2017) no.9, 094019 [arXiv:1707.03107 [hep-ph]].

[16] J. H. Sloan, Nucl. Phys. Proc. Suppl. 63 (1998) 365 [hep-lat/9710061].

R. R. Horgan et al., Phys. Rev. D 80 (2009) 074505 [arXiv:0906.0945 [hep-lat]]. 